\title{
BMJ Open Effect of a concussion on subsequent baseline SCAT performance in professional rugby players: a retrospective cohort study in global elite Rugby Union
}

\author{
Ross Tucker (D) ," Eanna Falvey, ${ }^{2,3}$ Gordon Fuller (D) , James Craig Brown, ${ }^{5}$ \\ Martin Raftery ${ }^{1}$
}

To cite: Tucker R, Falvey E, Fuller G, et al. Effect of a concussion on subsequent baseline SCAT performance in professional rugby players: a retrospective cohort study in global elite Rugby Union. BMJ Open 2020;10:e036894. doi:10.1136/ bmjopen-2020-036894

- Prepublication history for this paper is available online. To view these files, please visit the journal online (http://dx.doi. org/10.1136/bmjopen-2020036894).

Received 09 January 2020

Revised 09 June 2020

Accepted 25 June 2020

Check for updates

(C) Author(s) (or their employer(s)) 2020. Re-use permitted under CC BY-NC. No commercial re-use. See rights and permissions. Published by BMJ.

For numbered affiliations see end of article.

Correspondence to

Dr Ross Tucker;

Ross.tucker@mweb.co.za

\section{ABSTRACT}

Objectives This study assessed whether concussion affects subsequent baseline performance in professional rugby players. Annual baseline screening tests are used to guide return-to-play decisions and concussion diagnosis during subsequent screens. It is important that baseline performances are appropriate and valid for the duration of a season and unaffected by factors unrelated to the current head impact event. One such factor may be a concussion following baseline assessment.

Setting The World Rugby concussion management database for global professional Rugby Union.

Participants 501 professional rugby players with two baseline Sports Concussion Assessment Tools (SCATs) and an intervening concussion (CONC) were compared with 1190 control players with successive annual SCAT5s and no diagnosed concussion (CONT).

Primary and secondary outcome measures Symptom endorsement, cognitive and balance performance during annual SCAT baseline assessments.

Results Players with a diagnosed concussion (CONC) endorsed fewer symptoms (change $-0.42,95 \% \mathrm{Cl}-0.75$ to -0.09 ), and reported lower symptom severity scores during their second assessment $(T 2, p<0.001)$ than non-concussed players (CONT). Concussed players also improved Digits Backward and Final Concentration scores in T2 $(p<0.001)$. Tandem gait time was improved during T2 in CONT. No other sub-mode differences were observed in either group.

Conclusions Reduced symptom endorsement and improved cognitive performance after concussion may be the result of differences in the motivation of previously concussed players to avoid exclusion from play, leading to under-reporting of symptoms and greater effort in cognitive tests. Improved cognitive performance may be the result of familiarity with the tests as a result of greater exposure to concussion screening. The changes are small and unlikely to have clinical significance in most cases, though clinicians should be mindful of possible reasons, possibly repeating sub-modes and investigating players whose baseline scores change significantly after concussion. The findings do not necessitate a change in the sport's concussion management policy.

\section{Strengths and limitations of this study}

- This is a large-scale, real-life study that compares 501 professional men's Rugby Union players who experienced a concussion and who have a baseline Sports Concussion Assessment Tool (SCAT) assessment on either side of this head injury event, to 1190 professional men's players with two successive SCAT5s but without an intervening concussion.

- Data are obtained in a controlled environment, as per standardised assessments and an operational definition of concussion created by World Rugby.

- A limitation is that descriptive characteristics of players within the cohort, including age, ethnicity, playing experience and language, are unavailable.

- Study design (cross-sectional of a census sample) does not allow evaluation of the mechanism or factors responsible for observed changes between T2 and $\mathrm{T} 1$.

- Changes to the SCAT, including a version update affecting symptom reporting and a change in wordlength, reduce sample number for some sub-mode analyses.

\section{INTRODUCTION}

Concussion is the most prevalent injury in professional Rugby Union, ${ }^{1}$ with an incidence between 14 and 20 concussions per 1000 match hours. ${ }^{1-4}$ In most cases, cognitive deficits, balance and symptoms improve rapidly during the first 2 weeks after injury, resolving within 1 month, ${ }^{5}$ but in some cases, impairments may persist for longer, particularly with respects to symptoms. It has been found that previously concussed collegiate athletes report more symptoms at a greater severity than non-concussed players, ${ }^{6}$ with conflicting results on neurocognitive performance in college athletes, adolescents and adult jockeys. ${ }^{7-9}$ Should such concussion-related decrements persist beyond return-to-play, 
it may invalidate the baseline thresholds against which subsequent off-field and diagnostic screens are assessed.

It is important, therefore, that the baseline scores and clinical reference limits that are used to interpret abnormal scores for each sub-mode be appropriate and valid for the duration of a season, and unaffected by factors unrelated to the current head impact event. One factor that may directly affect a player's baseline performance is a concussion occurring after baseline measurement.

A concussion may also alter screening performance indirectly, through repeated exposure to tests and resultant learning effects. Rugby Union has used a three-point Head Injury Assessment (HIA) process since 2014. This process has been adapted from the Sports Concussion Assessment Tool (SCAT), and assesses a player at the time of head impact event during matches (HIA1), 3 hours after the match (HIA2) and after two nights' rest (HIA3). It is possible that players who undergo the process after head injuries become familiar with the symptoms, the questions asked during cognitive sub-modes and the performance of balance tests. Anecdotally, team doctors have occasionally reported players achieving better cognitive sub-mode scores during HIA2 or HIA3 testing than during baseline tests, along with reports that players learn cognitive sub-modes in order to pass diagnostic assessments. Collectively, a consideration is whether the diagnostic process after a concussion, consisting of the off-field screen, followed by the HIA2 and HIA3 assessments, create a learning effect in players who undergo those tests more frequently than non-concussed peers.

In either scenario, a previously obtained baseline assessment may be rendered invalid, either by persistent concussion-related impairments, or by learning effects owing to more frequent exposure to the screen submodes. Given that return to play and diagnostic decisions are made in comparison to a baseline SCAT, it is important to consider whether any changes are long-lived and large enough to compromise future clinical decisions. Assessing the degree and duration of potential changes in the SCAT5 sub-modes after a concussion will guide clinicians as to whether a player requires an updated baseline assessment after concussion, or whether the current requirement of annual baseline testing is sufficient.

\section{Aims}

The aim of the present study was to assess whether baseline SCAT performance is affected by previous concussion. Specifically, we explored how each SCAT sub-mode performance was affected by an intervening concussion, compared with a group of similarly matched players who did not experience a concussion between two successive annual baseline assessments. Since the control group comprised a group of players who underwent two SCAT5 baseline assessments but did not experience a concussion, we are also able to obtain a preliminary analysis of whether two repeated baselines influence performance, possibly through learning effects.

\section{METHODS}

\section{Study design, setting and study population}

A retrospective cohort study was performed using data from the World Rugby Head Injury Assessment (HIA) database, which contains baseline and diagnostic concussion screen results from the professional game. The HIA process used by Rugby Union is a three-phase protocol, adapted from the SCAT. The first phase combines an abridged SCAT (HIA1 off-field screen) conducted at the time of the head impact event with video review and clinical examinations to guide return to play decisions. After the game, full SCAT assessments within 3 hours of the match (HIA2 screen) and after two nights' rest (HIA3 screen) are used to support the diagnosis of concussion. ${ }^{10}$ During all three assessments, a player's performance in various sub-modes is assessed against their own previously performed SCAT5 baseline assessment, or if a baseline assessment is absent, against normative data (clinical reference limits) derived from a rugby-playing population. ${ }^{11}$

In order to use the HIA process, a competition must adhere to mandatory competition player welfare standards (World Rugby Player Welfare Site) that ensures a standardised approach to concussion detection and management as well as data collection. The source population thus comprises the majority of eligible professional male players in domestic and international competitions that underwent mandatory baseline SCAT assessment between 2015 and 2019.

\section{Patient and public involvement}

Patients or the public were not involved in the design, or conduct, or reporting, or dissemination plans of the research.

\section{Baseline screening}

Baseline SCAT assessments are typically administered before commencement of the relevant competition season or tournament, according to methods described previously. ${ }^{12}$ For the purposes of the present study, cases were identified as all professional players who had a baseline screen both before (CONC T1) and after (CONC T2) a documented concussion between 2017 and 2019. Concussions were identified as per the World Rugby operational definition, which requires either an abnormal HIA2 screen, HIA3 screen, a doctor diagnosis or the presence of a so-called Criteria 1 sign on-field at the time of the head impact event. ${ }^{1}$ Women were excluded from the analysis, since previous research has found greater symptom endorsement which may affect both the initial assessment and resultant changes. ${ }^{13}$ A control group (CONT) consisted of all professional male players with two SCAT5 baseline assessments but no documented concussions over the same sampling period (CONT T1 and CONT T2). Since we wished to explore only how a 'recent concussion' affected SCAT performance, we excluded cases where the T2 screen was conducted more than a year after the concussion, as well as baseline 
screens were done more than 2 years apart were excluded from analysis.

In some cases, the baseline screens at $\mathrm{T} 1$ and $\mathrm{T} 2$ were different versions of the SCAT, which changed from SCAT3 to SCAT5 during the sampling period, or used a different word length list. For our analysis of word-list sub-modes (Immediate memory and Delayed recall) and symptom endorsement, we analysed only paired and directly comparable sub-modes, and thus excluded all cases and controls where a sub-mode was assessed differently in T2 compared with T1. We report the number of available paired comparisons in the results. Since the sample was a fixed census sample, precision was indicated by the size of the CIs.

Raw scores were compared using descriptive statistics, while the change in each sub-mode was calculated as T2 minus T1 for CONC and CONT, and reported as mean change and $95 \%$ CIs. The proportion of players who improved, worsened and achieved the same score during T2 was determined for each group. An improvement in the sub-mode performance was identified when symptoms endorsed, errors made during balance tests and time taken to complete the tandem gait assessment reduced, whereas cognitive sub-modes were deemed to improve when their score increased in $\mathrm{T} 2$.

A Wilcoxon signed-rank test was performed to assess whether sub-mode scores were significantly different between $\mathrm{T} 2$ and $\mathrm{T} 1$ in CONC and CONT. The null hypothesis $(\mathrm{T} 2=\mathrm{T} 1)$ was rejected when $\mathrm{p}<0.004$, based on a Bonferroni correction of the original alpha of 0.05 , divided by the 12 SCAT subdomains assessed $(0.05 / 12=0.004)$.

The period between subsequent SCAT tests in both groups was assessed using a Mann-Whitney U test.

An ordinal regression model was developed to determine whether sub-mode performance changed differently in concussed players compared with controls. The change in sub-mode score (T2 minus T1) was categorised as 'worse' $(-1)$, 'no change' $(0)$ or 'improved' $(+1)$ as the dependent variable, and the number of days between tests used as an independent variable. Based on the fact that the period between tests was significantly different between the two groups $(p<0.001)$, days between tests was added as an independent variable to the logistic regression. Thus, an adjusted for time between tests OR was calculated for each sub-mode, reporting the odds of sub-mode performance improving more at T2 in CONC than at T2 in CONT. An OR greater than 1 and a $p$ value less than 0.0042 thus means that an improvement in submode performance in CONC T2 was more likely than in CONT T2. A likelihood ratio test was run after each regression that found a significant relationship in order to test the proportional odds assumption.

The influence of the period between the concussion and $\mathrm{T} 2$ in this group was examined by dividing the concussion cases into those players who had experienced a concussion within 3 months of the T2 baseline (recent, CONC REC), and players whose concussion occurred more than 3 months before the T2 assessment (distant, CONC DIST). REC and DIST were compared in terms of their distribution with the three outcome categories ('worsen', 'no change' and 'improve') using a Fisher's exact test. As there were 22 symptoms, the Bonferroni corrected $\mathrm{p}$ value for this Fisher's exact test was set at $0.0022(0.05 / 22)$ for these analyses.

\section{RESULTS}

501 players with a baseline SCAT assessment on each side of a diagnosed concussion were analysed for the CONC group. The median period between $\mathrm{T} 1$ and $\mathrm{T} 2$ baseline assessments was 362 days (IQR 318 to 395 days) and the period between the concussion and the T2 baseline screen was 222 days (IQR 139 to 295). The control cohort consisted of 1190 players who underwent two SCAT5 assessments without a concussion in the period between. Median period between T1 and T2 was 334 days (IQR 294 to 365 days).

The available paired cases for analysis, along with submode means, $95 \% \mathrm{CI}$ and medians are shown for $\mathrm{T} 1$ and $\mathrm{T} 2$ in the two groups in table 1. Mean Immediate memory score was greater in CONC than CONT at T1 and T2. Mean symptom number and severity were lower in CONC than CONT at T2, and Digits backward, Final concentration scores and Delayed Recall scores were higher in CONC than CONT at T2. No differences were found between median scores for any sub-mode at $\mathrm{T} 1$.

Table 2 shows the change in performance for each sub-mode, calculated as the difference between $\mathrm{T} 2$ and T1, whether the sub-mode score improved, worsened or remained the same compared with $\mathrm{T} 1$, and the proportion of players who fell into each category for that submode. The Wilcoxon signed-rank test $p$ value evaluates whether the T2 versus T1 change in that sub-mode was significant within each group. The OR evaluates whether an improvement in sub-mode score was more likely in the CONC than CONT group, with a ratio greater than 1 indicating that the sub-mode improved more in T2 of CONC than in T2 of CONT.

Figure 1 shows the changes in selected sub-modes for illustrative purposes, with symptoms (top left panel) and one sub-mode from cognitive (digits backward score, middle left panel) and one sub-mode from the balance (single leg errors, bottom left panel) sub-modes selected by virtue of being significantly different in CONC than CONT. Frequency histograms of sub-mode score changes for each of the three illustrative subdomains in the CONT group, the recent concussions (REC CONC, concussion within 3 months of POST) and distant concussions (DIST CONC, concussion more than 3 months before T2) are also shown.

\section{Symptoms}

Symptom number and severity were significantly lower at $\mathrm{T} 2$ than $\mathrm{T} 1$ in the CONC group $(\mathrm{p}=0.0022$ and $\mathrm{p}=0.001$ for number and severity, respectively), with $30.8 \%$ of 
Table 1 Descriptive statistics of sub-mode performance in players who experienced a concussion (CONC) and a control group with no concussions (CONT)

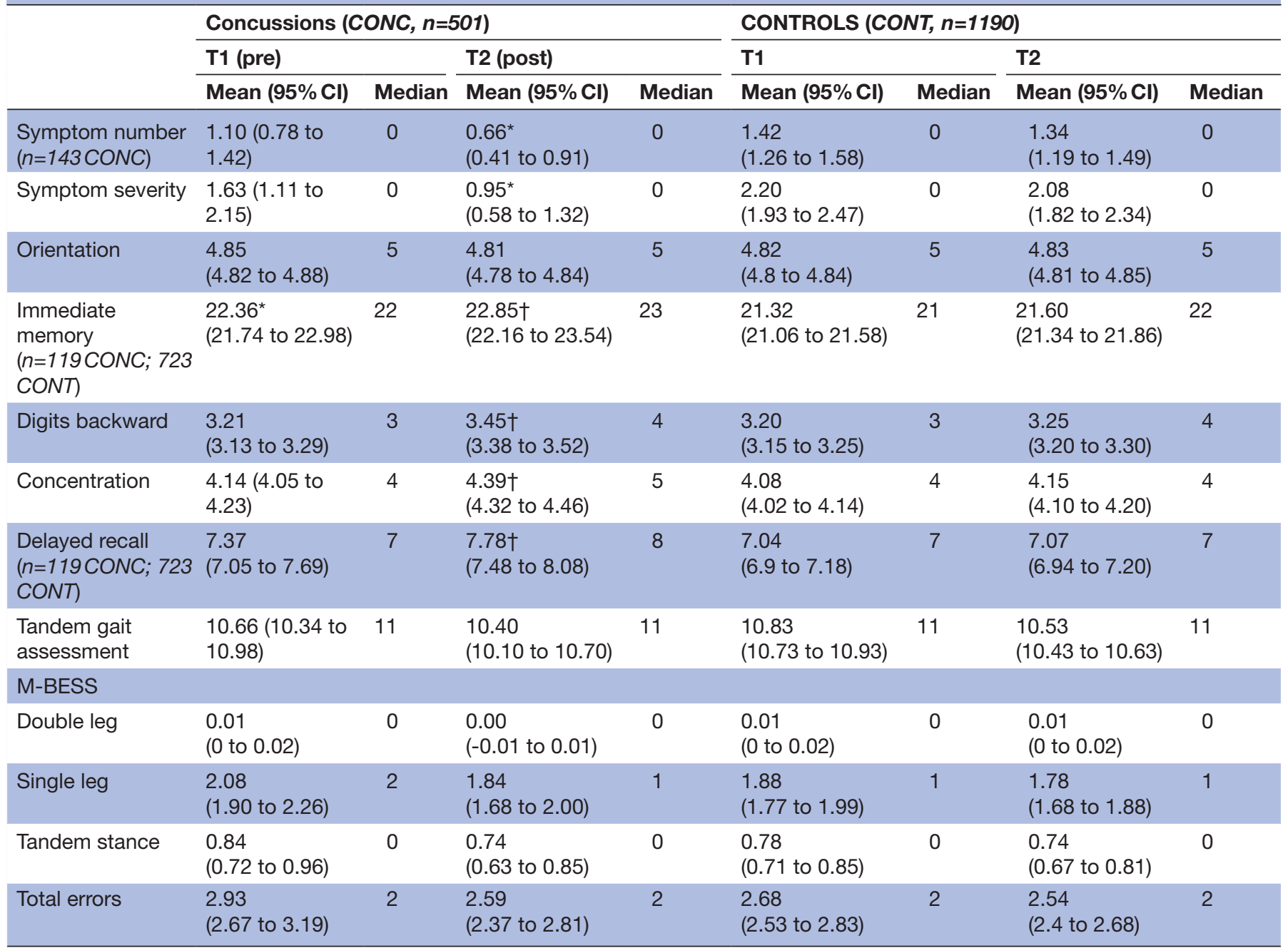

Sub-mode scores are shown as means $(95 \% \mathrm{Cl})$ and medians.

*denotes significantly lower than in CONT at the corresponding time point.

tdenotes significantly greater than in CONT at the corresponding time point

M-BESS, Modified Balance Error Scoring System.

players reporting fewer symptoms at T2, compared with $14.0 \%$ who endorsed more symptoms at T2 (table 2 and figure 1$)$. Of the players who reported fewer symptoms $(30.8 \%$, table 2$)$, almost half $(14.0 \%)$ decreased by only one symptom, while only $10 \%$ of players reduced symptom number by 3 or more. In the CONT group, symptom number and severity were similar between $\mathrm{T} 2$ and $\mathrm{T} 1$.

While there was a trend towards a decrease in symptom number and symptom severity in CONC compared with CONT (OR 1.52 (1.10 to 2.11, $\mathrm{p}=0.011$ ) for symptom number and for severity (OR 1.54 (1.11 to 2.13, $\mathrm{p}=0.009)$ ), this did not reach significance using the Bonferroni corrected $p$ value (table 2 ).

\section{Cognitive sub-modes}

Digits backward score was significantly better at T2 than T1 in CONC $(p<0.001)$ but was unchanged in CONT.
Final concentration score, of which Digits Backward makes up four of the five possible correct answers, was similarly improved in $\mathrm{T} 2$ compared with $\mathrm{T} 1$ in CONC, but was not different in the CONT group at T2 compared with T1.

Orientation scores, Immediate Memory and Delayed Recall performance were not different between $\mathrm{T} 2$ and T1 in either cohort (table 2).

\section{Balance sub-modes}

Tandem gait time was significantly faster in T2 compared with T1 (improvement of $0.29 \mathrm{~s}$, table 2 ) in CONT, but was not different in CONC $(\mathrm{p}=0.012)$. There was no difference in the odds of improvement in CONC and CONT.

Balance errors during Double, Single Leg and Tandem Stance were unchanged T2 versus T1 in both CONC and CONT. Total balance errors were thus unaffected by concussion and did not change over time in the CONT group. 


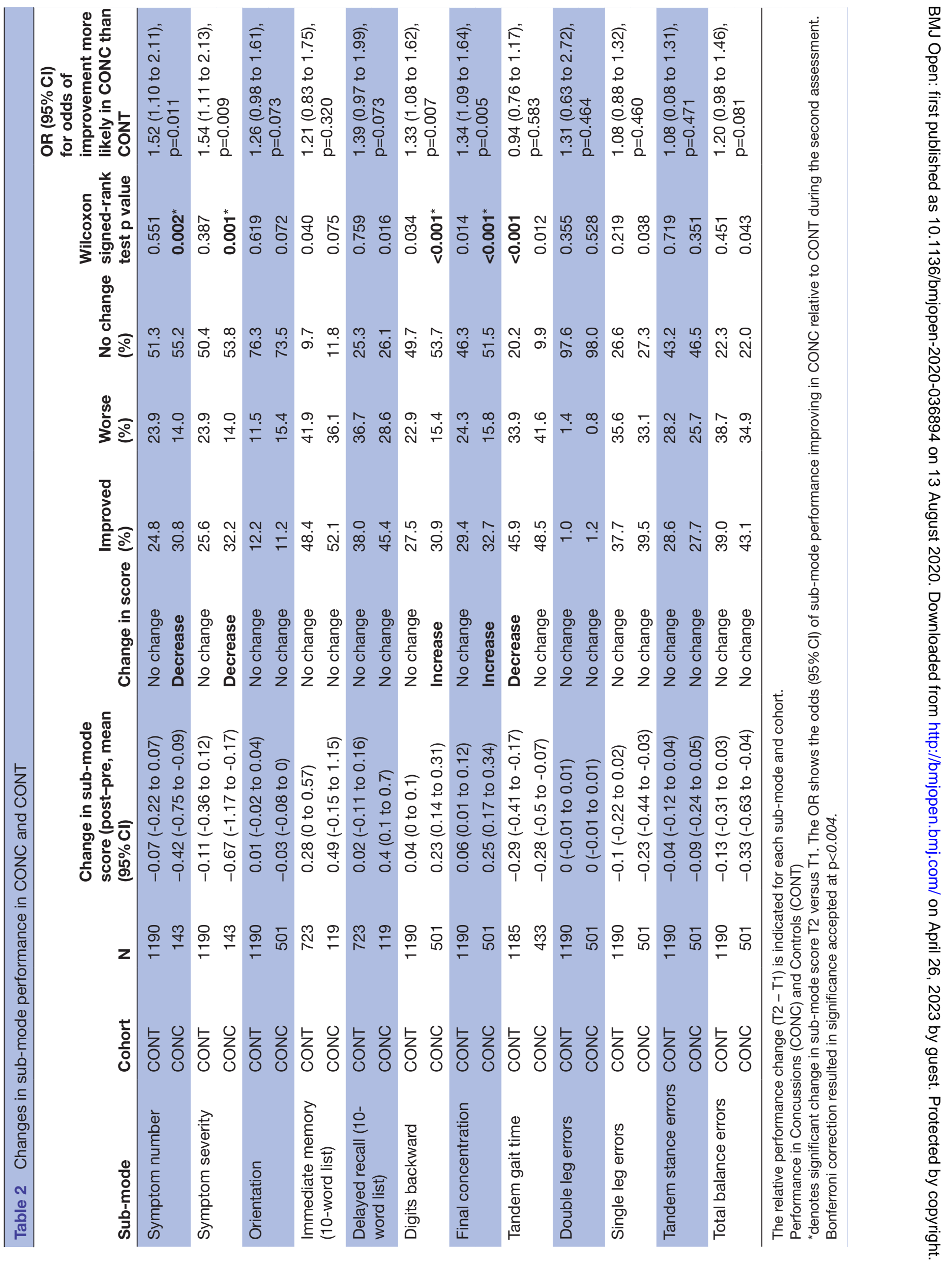



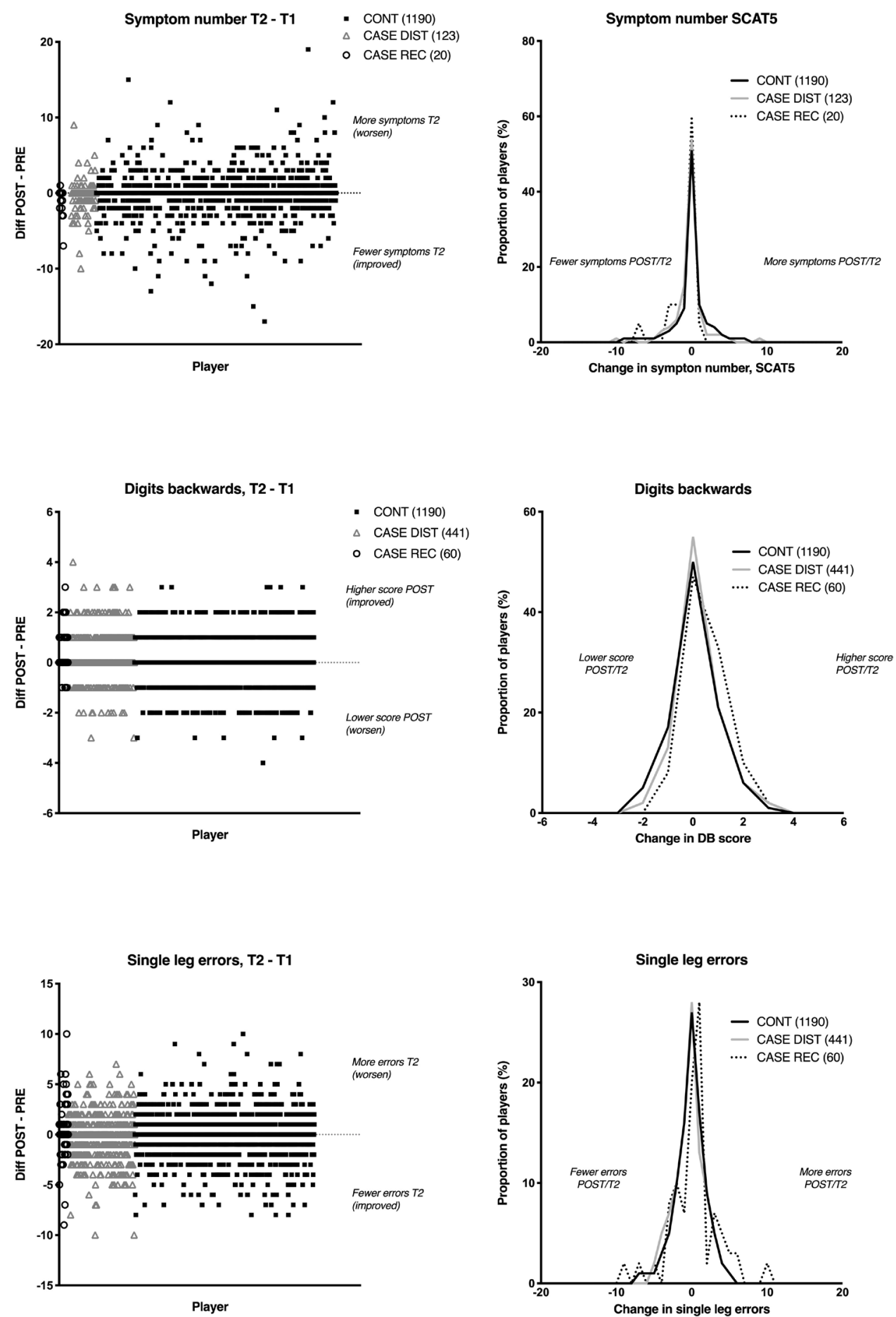

Figure 1 Changes in sub-mode performance (T2 - T1) for symptom number, digits backward and single leg errors (left column), and histograms depicting proportion of players within each cohort changing in the three selected sub-modes. Data are shown for all Controls (CONT) and Concussions (CONC) are divided into those where the concussion occurred more than 3 months before the T2 baseline (DIST) and fewer than 3 months before the T2 baseline (REC). SCAT,Sports Concussion Assessment Tool.

\section{Effect of time duration between concussion and second baseline test (post) gap on sub-mode performance}

The CONC cohort $(\mathrm{n}=501)$ was divided into players whose concussion occurred within 3 months of T2 baseline testing (REC, $\mathrm{n}=60$ ) and those whose concussion occurred more than 3 months before T1 baseline (DIST, $\mathrm{n}=441$ ). Table 3 shows the proportion of each group improving, worsening and staying the same in $\mathrm{T} 2$ versus $\mathrm{T} 1$, and the results of the $\chi^{2}$ analysis for each sub-mode.

No significant differences were found for any submode, with the proportion of players improving and worsening similar in REC and DIST concussion cases. A similar proportion of improved and worsened scores was found for Final Concentration score (table 3). 
Table $3 \chi^{2}$ analysis of improvements in SCAT5 sub-modes for recent ( $<3$ months) and distant ( $>3$ months) concussed players

\begin{tabular}{|c|c|c|c|c|c|c|}
\hline Sub-mode & Cohort & $\mathbf{N}$ & Improved (\%) & Worse (\%) & No change (\%) & $P$ value \\
\hline \multirow[t]{2}{*}{ Symptom number } & Distant & 123 & 30.1 & 15.4 & 54.5 & 0.548 \\
\hline & Recent & 20 & 35.0 & 5.0 & 60.0 & \\
\hline \multirow[t]{2}{*}{ Symptom severity } & Distant & 123 & 31.7 & 15.4 & 52.8 & 0.528 \\
\hline & Recent & 20 & 35.0 & 5.0 & 60.0 & \\
\hline \multirow[t]{2}{*}{ Orientation } & Distant & 441 & 10.7 & 15.2 & 74.1 & 0.501 \\
\hline & Recent & 60 & 15.0 & 16.7 & 68.3 & \\
\hline \multirow{2}{*}{$\begin{array}{l}\text { Immediate memory } \\
\text { (10-word list) }\end{array}$} & Distant & 101 & 52.5 & 36.6 & 10.9 & 0.819 \\
\hline & Recent & 18 & 50.0 & 33.3 & 16.7 & \\
\hline \multirow{2}{*}{$\begin{array}{l}\text { Delayed recall } \\
\text { (10-word list) }\end{array}$} & Distant & 101 & 44.6 & 27.7 & 27.7 & 0.644 \\
\hline & Recent & 18 & 50.0 & 33.3 & 16.7 & \\
\hline \multirow[t]{2}{*}{ Digits backward } & Distant & 441 & 29.0 & 16.3 & 54.6 & 0.032 \\
\hline & Recent & 60 & 45.0 & 8.3 & 46.7 & \\
\hline \multirow[t]{2}{*}{ Final concentration } & Distant & 441 & 30.6 & 16.6 & 52.8 & 0.026 \\
\hline & Recent & 60 & 48.3 & 10.0 & 41.7 & \\
\hline \multirow[t]{2}{*}{ Tandem gait time } & Distant & 381 & 49.3 & 40.9 & 9.7 & 0.602 \\
\hline & Recent & 52 & 42.3 & 46.2 & 11.5 & \\
\hline \multirow[t]{2}{*}{ Double leg errors } & Distant & 441 & 1.1 & 0.7 & 98.2 & 0.341 \\
\hline & Recent & 60 & 1.7 & 1.7 & 96.7 & \\
\hline \multirow[t]{2}{*}{ Single leg errors } & Distant & 441 & 40.8 & 30.8 & 28.3 & 0.016 \\
\hline & Recent & 60 & 30.0 & 50.0 & 20.0 & \\
\hline \multirow[t]{2}{*}{ Tandem stance errors } & Distant & 441 & 28.1 & 26.1 & 45.8 & 0.733 \\
\hline & Recent & 60 & 25.0 & 23.3 & 51.7 & \\
\hline \multirow[t]{2}{*}{ Total balance errors } & Distant & 441 & 43.8 & 33.1 & 23.1 & 0.051 \\
\hline & Recent & 60 & 38.3 & 48.3 & 13.3 & \\
\hline
\end{tabular}

Bonferroni correction resulted in significance accepted at $\mathrm{p}<0.002$.

SCAT, Sports Concussion Assessment Tool.

\section{DISCUSSION}

This study assessed whether SCAT5 sub-modes were altered in a group of professional rugby players who experienced a concussion, and whether this might have implications for the assessment of annual baseline screens and the clinical use and interpretation of return-to-play and diagnostic screens in the season after the concussion.

\section{Symptom endorsement after concussion}

Our first important finding was that players who experienced a concussion reported fewer symptoms during their T2 baseline screen than during T1 (table 2), with over twice as many players reporting fewer symptoms during T2. In contrast, there was no difference in symptom endorsement in $\mathrm{T} 2$ compared with $\mathrm{T} 1$ of the non-concussed control group.

The clinical significance of these changes is questionable, given the small size of the symptom number and severity reductions we document (table 2, figure 1). For example, while $30.8 \%$ of the concussed cohort reported fewer symptoms at $\mathrm{T} 2$, almost half of these players reported only one symptom fewer, and only $10.5 \%$ had a reduction of three or more symptoms compared with $\mathrm{T} 1$, a proportion similar to that observed in CONT (9.9\%). Given the non-specific nature of symptoms and their potential to vary from day to day for non-injury related reasons, and that clinicians are instructed to assess symptoms reported at the time of a baseline screen or a suspected head injury in the context of the player's normal symptom traits, these small changes are unlikely to influence clinical actions taken. This is particularly true given that recent research shows that clinicians frequently apply clinical judgement to overrule the presence of reported symptoms. ${ }^{14}$

The reduction in symptom endorsement we document contradicts research in college collision sport athletes, where a previous concussion was associated with greater baseline symptom endorsement and severity. ${ }^{6}$ Differences in study design, definitions and participant age and level may partly explain these differences. For example, previous studies have used self-reported concussion rather than a standardised clinical diagnosis of concussion. Given that athletes may under-report concussion symptoms, self-reporting may group concussed athletes 
with those who have truly never experienced a concussion. ${ }^{7}$ In our study, concussion was confirmed as part of the World Rugby operational definition for concussion, ${ }^{10}$ though it is still possible that players from our CONT group experienced concussions that were not detected at the time of injury.

The observed reduction in symptom endorsement in concussed players during T2 may be the result of familiarity with the concussion screening process, because these players are exposed to the SCAT more than nonconcussed players as part of World Rugby's HIA process. This may lead to these concussed players knowingly withholding symptoms that would exclude them from sporting participation in future. Desire to continue playing has been shown to be a primary motivator for under-reporting concussions. ${ }^{15}$ Players who experienced a previous concussion were less likely to report symptoms when assessed 2 hours after their next concussive event, ${ }^{6}$ suggested to be the result of less distress due to familiarity with the screen, as well as their deliberate minimisation of symptoms to return to play more quickly, having experienced a concussion-induced absence previously. ${ }^{6}$ In the short-term (first 2 weeks) after concussion, male athletes have been shown to report more symptoms than female athletes, a difference attributed to male players' desire to avoid removal from sport. ${ }^{16}$

It may therefore be that players who suffer concussions become more familiar with the screening and clinical process and recognise that their symptom endorsement increases the amount of time they may remain on the side-line in the event of a future concussion. While the time period between injury and reporting is considerably greater in this study than previous research, it is possible that players continue to knowingly downplay any symptoms when they are assessed prior to their next season.

We attempted to explore this possibility by analysing a sub-cohort of players who had experienced their concussion within 3 months of T2, but found no significant effects in this analysis (table 3). This is likely due to a small sample size for recent concussions, since we did find tendencies for changes in symptom endorsement after more recent concussions, sufficient to hypothesise that the time between injury and screen may be a relevant factor. This is an area that should be considered for future research.

\section{Cognitive sub-modes}

Our second important finding was that most cognitive submodes are unaffected by concussion, and that no cognitive sub-modes change in the CONT group. We found a significant improvement in Digits Backward score in T2 in CONC, and thus improved Final Concentration score, of which Digits Backwards comprises four of the five available correct answers (table 2, figure 1). Mean Delayed recall score was significantly higher at $\mathrm{T} 2$ in concussed players (table 1), but no statistical difference was found in the change from $\mathrm{T} 1$ to $\mathrm{T} 2$, or the odds of an improvement in CONC relative to CONT.
We suggest that this may be the result of test exposure, motivation to perform better after a concussion, and memory, which may be related to the period between concussion testing and repeat baseline, since we found that a higher proportion of the 60 REC cases improved on concentration testing compared with DIST cases (table 3).

Previous research supports this possibility, since previously concussed college athletes have been found to score better than never-concussed athletes in most cognitive sub-modes of the SCAT3. ${ }^{17}$ This was attributed either to effort differences between concussed and non-concussed players, or a learning effect as a result of repeated exposures to the tests during diagnostic and return-to-play protocols.

Other research has found no effects of distantlyoccurring concussion on cognitive function, assessed using computerised or neurocognitive tests, ${ }^{7}$ while others have found detrimental effects of distant concussions on neuropsychological assessments in jockeys ${ }^{8}$ and high school athletes. ${ }^{9} 18$ Differences between these findings and the present study may exist as a result of severity of concussions being greater among jockeys, the age of the players assessed, the time period between the concussion and the neurocognitive assessment and self-reported versus clinically diagnosed concussions as inclusion criteria, as described by Bruce and Echemendia. ${ }^{7}$

In the present study, since the CONT group performed only a single assessment approximately 1 year apart, compared with multiple assessments (HIA1, HIA2 and HIA3) with the cognitive sub-modes in the CONC group, it seems reasonable to suggest that repeated exposure to testing, combined with a possible desire to perform well in the cognitive sub-modes after concussion, improves performance through both learning and effort mechanisms. This effect was not found for Immediate Memory, which may reflect the greater challenge of using the 10-word lists in SCAT5 screens.

The implication of this finding is that annual baseline screening is an important requirement for cognitive submodes, to ensure that each player's baseline reference scores are updated to account for possible learning from more frequent testing. The paradox is that repeating the screen may be a contributing factor to improved scores, and so clinicians are advised to vary the word or number sequence within each cognitive sub-mode, particularly if a player presents with improved scores compared with previous screens. Our finding also reinforces that the tests used to assess cognitive function should be varied to prevent learning, and potentially increased in difficulty to eliminate ceiling effects and the ease with which words or number sequences can be memorised.

\section{Balance sub-modes and concussion}

We found that tandem gait time improved in $\mathrm{T} 2$ in the CONT group, but not in CONC. The improvement observed was $0.29 \mathrm{~s}(0.17$ to $0.41 \mathrm{~s})$, though there was no significant difference in the odds of a CONT player 
improving compared with previously concussed player (table 2), and the proportion of players in each group whose performance changed by more than one second was similar.

Other balance sub-modes were similar between CONT and CONC. We did find a tendency for a greater proportion of players to make more single leg errors in REC cases $(50 \%$ worse, compared with $30 \%$ with fewer errors, $\mathrm{n}=60)$ compared with DIST cases $(30.8 \%$ more errors compared with $40.8 \%$ making fewer errors, table 3 ). This could either be the result of the direct effects of concussive injury on balance in the more recent aftermath of concussion, or may be related to detraining after the concussive injury, but further research may be required to explore this.

It may be significant that balance sub-modes, unlike symptom endorsement or cognitive performance, can be neither learnt, practiced nor biassed by subjective reporting. The absence of differences in T2 of CONC, with the exception of small impairments in tandem gait time and single leg balance after more recent concussions, may reflect a more objective standard for the evaluation of lasting effects of concussion, though the precise manifestation of brain injury on various sub-modes is complex.

Given that a previous concussion does not affect balance scores significantly, and that we do not detect balance improvements as a result of repeat testing, it may be considered that annual screening of balance sub-modes is not necessary. However, we advise caution before any changes are made to current process, since we cannot assure that more recent concussions do not present with affected balance, or how repeated concussions may alter balance performance. As such, until further evidence confirms no effect on balance, we would advise that balance should be assessed as part of annual screening.

\section{Limitations}

Our analysis compared SCAT5 performance in a concussed group to a control group who underwent two SCAT5 assessments approximately 1 year apart, with no documented concussion between them. It is possible that a concussion or head injury was undiagnosed during this period, which may affect players in CONT, and possibly prior to T1 SCAT5 in both groups. The relative stability of testing both after concussion and in the control group means that though this cannot be excluded, it is unlikely to materially affect the results.

We also did not exclude players who would have performed multiple SCATs prior to the analysis period in this study, whether in the form of the off-field screen, diagnostic screens or baseline assessments. Our cohort included players with between zero and three SCAT assessments prior to their T1 assessment in the present study, though the proportion of players with these prior tests was similar between CONC and CONT. For this study, we chose to include players irrespective of previous SCAT history, because the formalised use of the SCAT5 in rugby means that all players in their second season or more of rugby who undergo the diagnostic and baseline are going to be exposed to repeated testing. Thus, for the sake of external validity of our findings, we felt it preferred to include all players, irrespective of their previous SCAT history.

The study design does not allow us to quantify the magnitude of any possible learning effects, in part because of the range of factors that may affect test performance, such as time between tests, time between concussion and T2 screens, language, ethnicity, age and fitness levels both pre-injury and post-injury, all of which have been found to affect performance during cognitive performance and symptom endorsement. ${ }^{19-23}$ As such, we can identify and postulate that possible learning effects explain the changes in performance in T2 versus T1, but we cannot quantify the magnitude of these changes distinct from normal test to test variation, and this would require a specific study to assess. Similarly, we are unable to account for possible differences in characteristics between the CASE and CONT group in the present study. Any differences, which may include age, playing level, experience, ethnic group and language, could influence differences between the groups.

Finally, the study design used here does not allow us to commit to a change in sub-modes score that would always have clinical meaning. For all the above-mentioned limitations, future research may explore how multiple tests, rather than two tests, influence performance as a result of potential learning effects. This research should assess players who are known not to have been concussed, undertaking tests with the same frequency as would typically occur in concussed players and then compared with concussed players to quantify the magnitude of learning effects and the variation in sub-mode scores that is clinically meaningful.

\section{Clinical implications}

We have described that symptom endorsement during baseline assessments is reduced in players with a previous concussion, that digits backwards and final concentration scores are improved during a baseline screen after a concussion, and that single leg balance performance may be impaired when baseline assessments are done within 3 months of the concussive event. We do not believe that these changes are clinically meaningful, given the small changes we observed, and the non-specificity and lack of precision of the sub-modes.

With respect to symptoms, given the small reduction in symptom endorsement, and that symptoms endorsed during SCAT5 screens are assessed relative to a player's trait symptoms, ${ }^{17}$ our finding does not meaningfully alter the practice of annual baseline tests, or necessitate a policy change for the management of previously concussed players during baseline or diagnostic screens. Clinicians should however be mindful of the possibility that previously concussed players may deliberately report fewer symptoms, and investigate the basis of any reductions to symptom number or severity. This may take the form of 
repeating the screen on different day, or a more in-depth consultation with the player regarding symptoms.

The improvements in cognitive sub-modes were small (0.23 improvement in Digits backward, for example, table 2), and unlikely to influence clinical decisions and actions at the time of suspected head injury events. This is particularly true given that it has recently been shown that doctors frequently apply clinical judgement to abnormal sub-modes during return to play and diagnostic screens. ${ }^{14}$ As such, the small changes we document in symptoms, digits backwards scores and tandem gait time do not necessitate a change in baseline screening policy, or use of baseline or diagnostic screens.

The findings are however important to guide clinicians in their assessment of changes in sub-mode performance. During baseline screens, clinicians should be mindful that reduced symptoms or improved cognitive scores may indicate either learning effects, greater motivation or an unwillingness to disclose symptoms that could prejudice a player's selection for teams. In this regard, changes to baseline performance should be assessed and interpreted with caution when a player has experienced a previous concussion. This may involve follow-up investigation and the repetition of the sub-modes in questions, using different word or number sequences, and in-depth consultation on symptoms where relevant.

Further research is required to explore whether multiple testing, consisting of three or more SCAT assessments, produces learning effects and to what degree submode performance may change even in the absence of concussions.

\section{Author affiliations}

${ }^{1}$ World Rugby Pty Ltd, Dublin, Ireland

${ }^{2}$ Department of Sports Medicine, Sports Surgery Clinic, Dublin, Ireland

${ }^{3}$ Department of Medicine, University College Cork, Cork, Ireland

${ }^{4}$ School of Health and Related Research, University of Sheffield Section of Public Health, Sheffield, UK

${ }^{5}$ Institute of Sport and Exercise Medicine, Department of Orthopaedics, Stellenbosch University, Cape Town, Western Cape, South Africa

\section{Twitter Ross Tucker @scienceofsport}

Acknowledgements We would like to acknowledge and sincerely thank the team doctors and medical practitioners for their help in facilitating collection of Sports Concussion Assessment Tool (SCAT) data.

Contributors MR conceived the study. MR, RT and EF designed the study. RT and JB performed the analyses. All authors (RT, EF, JB and GF) made substantial contributions to the study design, data processing and interpretation. RT drafted the article and all other authors revised it critically for important intellectual content. RT is the guarantor. All authors had full access to all of the data in the study and can take responsibility for the integrity of the data and the accuracy of the data analysis.

Funding There was no specific funding for the research study, though the research was supported by World Rugby, which employs some of the author group, as described in Competing Interests.

Competing interests Two of the authors (RT and EF) are employed full-time and part-time by World Rugby in roles of research and medicine. GF has served as an independent advisor on a working group on concussion administered by World Rugby, for which expenses are covered.

Patient consent for publication Not required.

Ethics approval The research plan for this study was approved by the World Rugby Institutional Ethics committee (REF 19007). Players had provided written informed consent for all data gathered as part of the World Rugby Concussion management programme to be used for research in a de-identified manner.

Provenance and peer review Not commissioned; externally peer reviewed.

Data availability statement Data are available upon reasonable request. Original participant data belongs to the players and the clubs/unions that generate such data. This may be provided upon request to third parties. World Rugby (the corresponding author) may facilitate the provision of that data, in terms of permissions and contacts, though there is not a single point of contact, since the data are generated globally from multiple teams and Unions.

Open access This is an open access article distributed in accordance with the Creative Commons Attribution Non Commercial (CC BY-NC 4.0) license, which permits others to distribute, remix, adapt, build upon this work non-commercially, and license their derivative works on different terms, provided the original work is properly cited, appropriate credit is given, any changes made indicated, and the use is non-commercial. See: http://creativecommons.org/licenses/by-nc/4.0/.

\section{ORCID iDs}

Ross Tucker http://orcid.org/0000-0002-4294-4822

Gordon Fuller http://orcid.org/0000-0001-8532-3500

\section{REFERENCES}

1 Fuller CW, Taylor A, Kemp SPT, et al. Rugby world cup 2015: world rugby injury surveillance study. Br J Sports Med 2017;51:51-7.

2 Fuller CW, Fuller GW, Kemp SPT, et al. Evaluation of World rugby's concussion management process: results from rugby world cup 2015. Br J Sports Med 2017;51:64-9.

3 Tucker R, Raftery M, Fuller GW, et al. A video analysis of head injuries satisfying the criteria for a head injury assessment in professional rugby Union: a prospective cohort study. $\mathrm{Br} J$ Sports Med 2017; 51:1147-51.

4 Tucker R, Raftery M, Kemp S, et al. Risk factors for head injury events in professional rugby Union: a video analysis of 464 head injury events to inform proposed injury prevention strategies. $\mathrm{Br} \mathrm{J}$ Sports Med 2017;51:1152-7.

5 McCrory P, Meeuwisse W, Dvořák J, et al. Consensus statement on concussion in sport-the $5^{\text {th }}$ international conference on concussion in sport held in Berlin, October 2016. Br J Sports Med 2017;51:838-47.

6 Bruce JM, Echemendia RJ. Concussion history predicts self-reported symptoms before and following a concussive event. Neurology 2004;63:1516-8.

7 Bruce JM, Echemendia RJ. History of multiple self-reported concussions is not associated with reduced cognitive abilities. Neurosurgery 2009;64:100-6. discussion106.

8 Wall SE, Williams WH, Cartwright-Hatton S, et al. Neuropsychological dysfunction following repeat concussions in jockeys. J Neurol Neurosurg Psychiatry 2006;77:518-20.

9 Moser RS, Schatz P, Jordan BD. Prolonged effects of concussion in high school athletes. Neurosurgery 2005;57:300-6. discussion300-6.

10 Raftery M, Kemp S, Patricios J, et al. It is time to give concussion an operational definition: a 3-step process to diagnose (or rule out) concussion within $48 \mathrm{~h}$ of injury: World Rugby guideline. $\mathrm{Br} J$ Sports Med 2016;50:642-3.

11 Fuller GW, Raftery M. Sport concussion assessment ToolFifth edition normative reference values for professional rugby union players. Clin J Sport Med 2018. doi:10.1097/ JSM.0000000000000713. [Epub ahead of print: 24 Dec 2018].

12 Fuller GW, Govind O, Tucker R, et al. Sport concussion assessment tool-Third edition normative reference values for professional rugby union players. J Sci Med Sport 2018;21:347-51.

13 Covassin T, Swanik CB, Sachs M, et al. Sex differences in baseline neuropsychological function and concussion symptoms of collegiate athletes. Br J Sports Med 2006;40:923-7. discussion927.

14 Fuller GW, Tucker R, Starling L, et al. The performance of the world rugby head injury assessment screening tool: a diagnostic accuracy study. Sports Med Open 2020;6:2.

15 Kerr ZY, Register-Mihalik JK, Kroshus E, et al. Motivations associated with nondisclosure of self-reported concussions in former collegiate athletes. Am J Sports Med 2016;44:220-5.

16 Covassin T, Elbin RJ, Harris W, et al. The role of age and sex in symptoms, neurocognitive performance, and postural stability in athletes after concussion. Am J Sports Med 2012;40:1303-12.

17 Shehata N, Wiley JP, Richea S, et al. Sport concussion assessment tool: baseline values for varsity collision sport athletes. $\mathrm{Br} J$ Sports Med 2009;43:730-4. 
18 Moser RS, Schatz P. Enduring effects of concussion in youth athletes. Arch Clin Neuropsychol 2002;17:91-100.

19 Norheim N, Kissinger-Knox A, Cheatham M, et al. Performance of college athletes on the 10-item word list of SCAT5. BMJ Open Sport Exerc Med 2018;4:e000412.

20 Asken BM, Houck ZM, Bauer RM, et al. SCAT5 vs. SCAT3 symptom reporting differences and convergent validity in collegiate athletes. Arch Clin Neuropsychol 2020;35:963-11.
21 Jinguji TM, Bompadre V, Harmon KG, et al. Sport concussion assessment Tool-2: baseline values for high school athletes. $\mathrm{Br} \mathrm{J}$ Sports Med 2012;46:365-70.

22 Mrazik M, Naidu D, Lebrun C, et al. Does an individual's fitness level affect baseline concussion symptoms? J Athl Train 2013;48:654-8.

23 Covassin T, Elbin RJ, Larson E, et al. Sex and age differences in depression and baseline sport-related concussion neurocognitive performance and symptoms. Clin J Sport Med 2012;22:98-104. 